\title{
Electrical Energy Harvesting from the Flexible Liquid Crystal Cells
}

\author{
Jae Hoon Lee ${ }^{a}$, Jun-Yong Lee ${ }^{a}$, Jeong-Seon Yub , Jong-Hyun Kima,b,* \\ aDepartment of Physics, Chungnam National University, Daejeon, 34134, Korea \\ 'Institute of Quantum Systems, Chungnam National University, Daejeon, 34134, Korea, \\ *jxk97@cnu.ac.kr
}

Supporting Information

Figure S1 shows the LC texture of the vertically aligned cells. However, in practice, the PET used as a substrate has an anisotropic refractive index according to the direction. Therefore, even two substrates without LC can change the brightness according to the direction in orthogonal polarizers. Considering this, the changes shown in S. 1 indicate that the cell is inducing a sufficient vertical alignment.
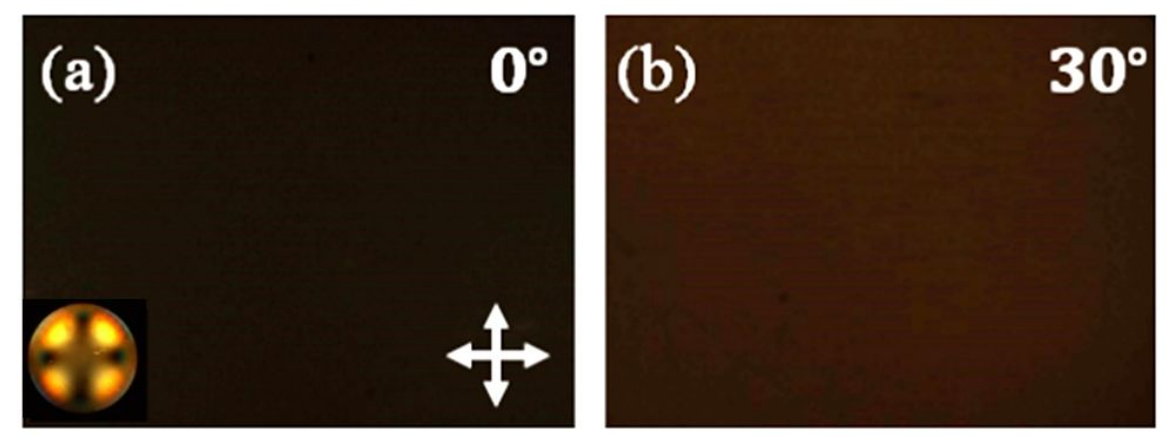

S1. Texture images measured using a polarizing optical microscope while rotating the vertical aligned cell. The polarizers are perpendicular to each other. (b) is rotated images from the image of (a). We assign $0^{\circ}$ as the direction of the substrate adjusted to make it darkest. The small box in (a) is a conoscopic image. The overall brightness was enhanced to clarify the image difference. Arrows indicate the direction of the polarizers. The size of the picture is $1.6 \mathrm{~mm}$ on the left and right. 
The theoretical and experimental values of bending is shown in Figure S2 and they agree relatively well in the experimental range.

(a)

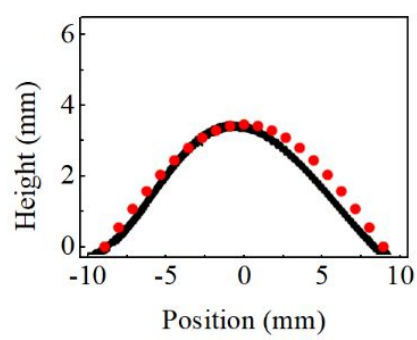

(b)

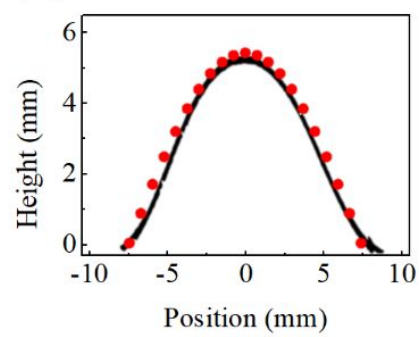

S2. Image viewed from the front when holding substrates at both ends and reducing the distance. The displacement (reduced length) is (a) $2 \mathrm{~mm}$ and (b) $5 \mathrm{~mm}$, respectively. The black solid line represents the experimental result, and the red dotted line represents the calculation. Height 0 is the reference of height. In the experiment, the cell exhibits with negligible irregularity at the parts that hold the cell. The cell length is $20 \mathrm{~mm}$ without bending. 